\title{
Absolute Calibration of Mid to Large Size IACTs with P1/P2 of the Crab Pulsar
}

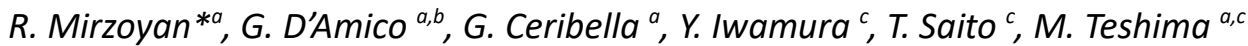 \\ ${ }^{a}$ Max-Planck-Institute for Physics, \\ Foehringer Ring 6, Munich, Germany \\ ${ }^{b}$ Department for Physics and Technology, \\ University of Bergen, Bergen NO-5020, Norway \\ ${ }^{c}$ University of Tokyo, ICRR \\ Tokyo, Japan \\ E-mail: Razmik.Mirzoyan@mpp.mpg.de, damico@mpp.mpg.de, \\ ceribell@mpp.mpg.de
}

\begin{abstract}
Mid to large-size imaging atmospheric Cherenkov telescopes for gamma-ray astrophysics have a typical threshold of $\sim(20-100) \mathrm{GeV}$ in the very high energy domain. In this energy range sensitive observations of the Crab Nebula can reveal the emission from the Crab pulsar at phases P1 and P2. Observations of the Crab pulsar by the MAGIC telescopes show that the $\mathrm{P} 2 / \mathrm{P} 1$ is a monotonically increasing function of energy in some certain range of energy. In a few tens of GeV energy range MAGIC sensitivity overlaps with that of the Fermi LAT mission, which was absolutely calibrated before the launch as well as keeps monitoring it during the flight. Comparison of the P2/P1 ratio from the MAGIC and Fermi LAT Crab pulsar data allows one to cross-calibrate the two instruments. Here we suggest using the P2/P1 ratio from the Crab pulsar observations for absolutely calibrating the operational energy range of mid to large-size IACTs.
\end{abstract}

37th International Cosmic Ray Conference (ICRC 2021)

July 12th-23rd, 2021

Online - Berlin, Germany 


\section{Introduction}

In the TeraelectronVolt $(\mathrm{TeV})$ energy range the Crab Nebula is still considered as the standard candle for calibrating instruments. It is considered as a steady, strongest source of $\mathrm{TeV}$ gamma rays in our galaxy. Since about $\sim 10$ years we know that the situation is different at lower, 100's of $\mathrm{MeV}$ to few $\mathrm{GeV}$ energy range, where from time to time the source flares, sometimes with the amplitude exceeding the base level by a few tens of times [1,2]. Attempts to observe the flares in the 100's of $\mathrm{TeV}$ energy, possibly due to Inverse Compton scattering effect, yet were not successful [3].

When a new instrument starts operating in the $\mathrm{TeV}$ energy range $(20 \mathrm{GeV}-100 \mathrm{TeV})$, one of the first things is to measure a signal from the Crab Nebula. By simulating the response of a given instrument to the Crab-like spectrum in the given energy range and comparing with the experimentally observed rate of gamma-rays, one can estimate its energy threshold and link event rates to the energy scale. Comparison with Monte Carlo simulations is of key importance for such measurement.

Often the comparison of results measured by one instrument with another one, operating in the overlapping energy range, is not straightforward, especially if the basic operational principles of the instruments are different. For example, one measures Cherenkov light emission from gamma-ray induced extensive air showers (EAS) in atmosphere (by an Imaging Air Cherenkov Telescope), while the other one measures light emission in (kept in the dark) scintillation particle detectors or water tanks or pools (like LHAASO or HAWC) or by converting gamma-rays into $\mathrm{e}^{ \pm}$pairs and measuring their parameters (like in Fermi LAT mission). Often the researchers use the same or a very similar Monte Carlo simulation codes, but some differences remain due to sometimes approximate or idealized introduction of instrumental parameters and resolutions. These can be, for example, the reduced reflectance of the used for several years mirrors and/or the quantum efficiency and photo electron collection efficiency, the noise factor $\mathrm{F}$ of classical photo-multiplier tubes and/or the photon detection efficiency of SiPM at the operational applied voltage, etc.

It could be an advantage to inter-calibrate different instruments by a simple method, which does not depend on extensive use of Monte Carlo simulations.

\section{The very high energy $\gamma$-ray emission of the Crab pulsar at phases P1 and P2}

The Crab pulsar is the power dynamo of the Crab Nebula. Its gamma-ray spectrum has been measured by the EGRET instrument of Compton Gamma Ray Observatory (CGRO) mission till the energy of $\sim 10 \mathrm{GeV}$ with an estimated cutoff at $\sim 6 \mathrm{GeV}$ [4]. The measurement of a $2.9 \sigma$ weak hint of gamma-rays with energy above $60 \mathrm{GeV}$ [5] was just a curious result by MAGIC. Very soon the same instrument revealed a clear signal above $25 \mathrm{GeV}$ at the phases P1 and P2 [6]. This made a strong impact, canceling out some models while questioning viability of the other ones. This was followed by the measurement of the pulsar spectrum till the energy $\sim 100 \mathrm{GeV}$ [7], Veritas in the energy range 120-250 GeV [8], MAGIC till $400 \mathrm{GeV}$ [9]. The latter showed the power-law behavior of the joint Fermi-LAT-MAGIC spectrum for energies 
above $\sim 10 \mathrm{GeV}$, which could be interpreted as evidence of the Inverse Compton (IC) emission mechanism at work. In the meantime, also the so-called "bridge" emission between the P1 and P2 has been discovered till the energy of $\sim 200 \mathrm{GeV}$ by MAGIC [10]. Another interesting publication from MAGIC showed that the pulsar spectrum extends till the energy of $\sim 1.2 \mathrm{TeV}$ for P2 and $\sim 600 \mathrm{GeV}$ for P1, with no sign of cutoff [11]. It is interesting to look at Figure 1, where one can see the SED of the P1 and P2 emission phases.

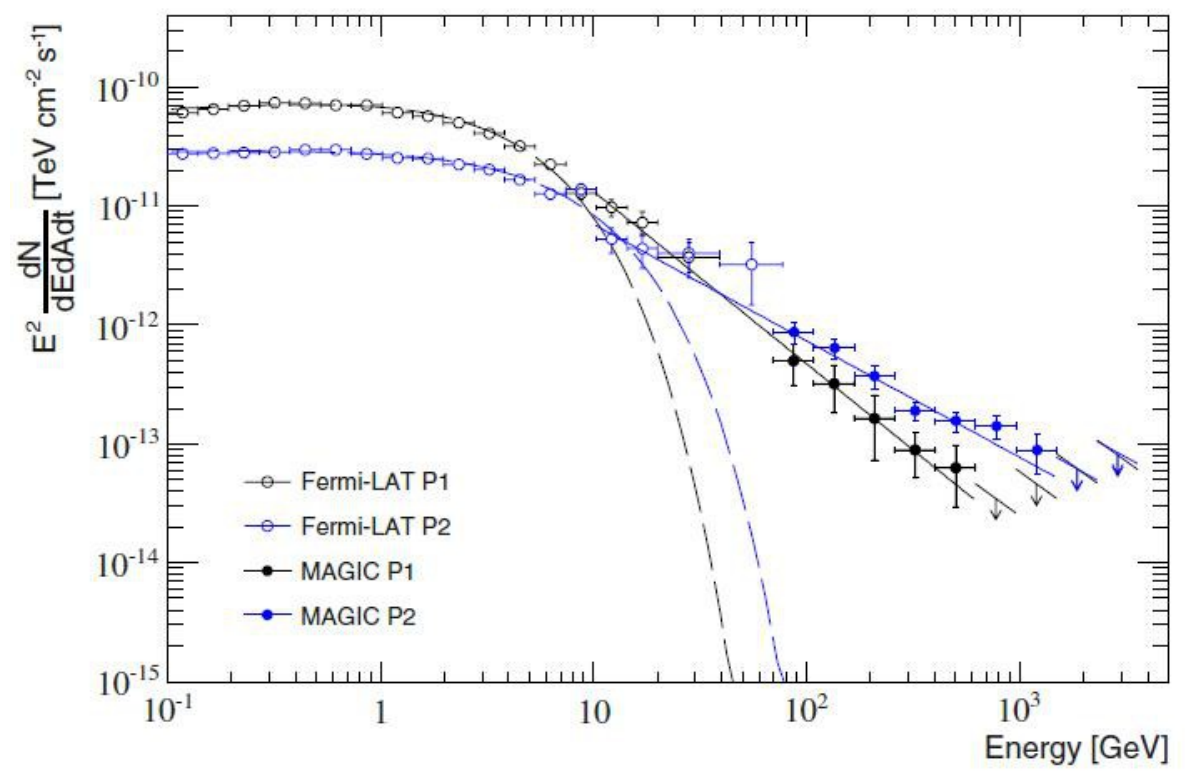

Fig.1. Phase-folded SED of the Crab P1 (black circles) and P2 (blue circles) at HE and VHE (open and filled circles). The results of the power law with exponential cutoff fits to the Fermi-LAT points are shown by dashed lines [10], whereas the joint Fermi-LAT/MAGIC fits to power-law functions above $10 \mathrm{GeV}$ are shown by solid lines. Image taken from [11].

The measured SED extends from $\sim 100 \mathrm{MeV}$ till $\sim 1.2 \mathrm{TeV}$. The spectrum measured by Fermi LAT at lower energies extends to the measured by MAGIC higher energies. Note that the SED of P1 and P2 curves become comparable (cross each other) at the energy of $\sim 40 \mathrm{GeV}$.

Let us assume the shown in Table.1 values for the intrinsic flux of the Crab pulsar at phases $\mathrm{P} 1$ and $\mathrm{P} 2$.

\begin{tabular}{|c|c|c|c|c|}
\hline \multicolumn{5}{|c|}{$\mathcal{F}(\boldsymbol{E})=\boldsymbol{f}_{\mathbf{0}}\left(\boldsymbol{E} / \boldsymbol{E}_{\mathbf{0}}\right)^{-\boldsymbol{\Gamma}}$} \\
\hline \hline Pulse & $\boldsymbol{E}_{\mathbf{0}}$ & $\boldsymbol{f}_{\mathbf{0}}$ & $\boldsymbol{\Gamma}$ & $\boldsymbol{\chi}^{\mathbf{2}} / \mathrm{NDF}$ \\
\hline P1 & 59.86 & $(3.83 \pm 0.42) \cdot 10^{-10}$ & $3.49 \pm 0.25$ & $4.6 / 3$ \\
\hline P2 & 70.33 & $(3.66 \pm 0.23) \cdot 10^{-10}$ & $3.18 \pm 0.11$ & $4.0 / 5$ \\
\hline
\end{tabular}

Table 1. The assumed intrinsic flux of the Crab pulsar at phases P1 and P2, taken from [12]. The spectra were obtained from the MAGIC data with a forward folding procedure with a power-law function. Energy is in units of GeV and the flux in $\mathrm{TeV}^{-1} \cdot \mathrm{cm}^{-2} \cdot \mathrm{s}^{-1}$.

The corresponding evolution in energy of the number of detected gammas from the Crab pulsar at phases P1 and P2 spectra are shown on Figure 2. 


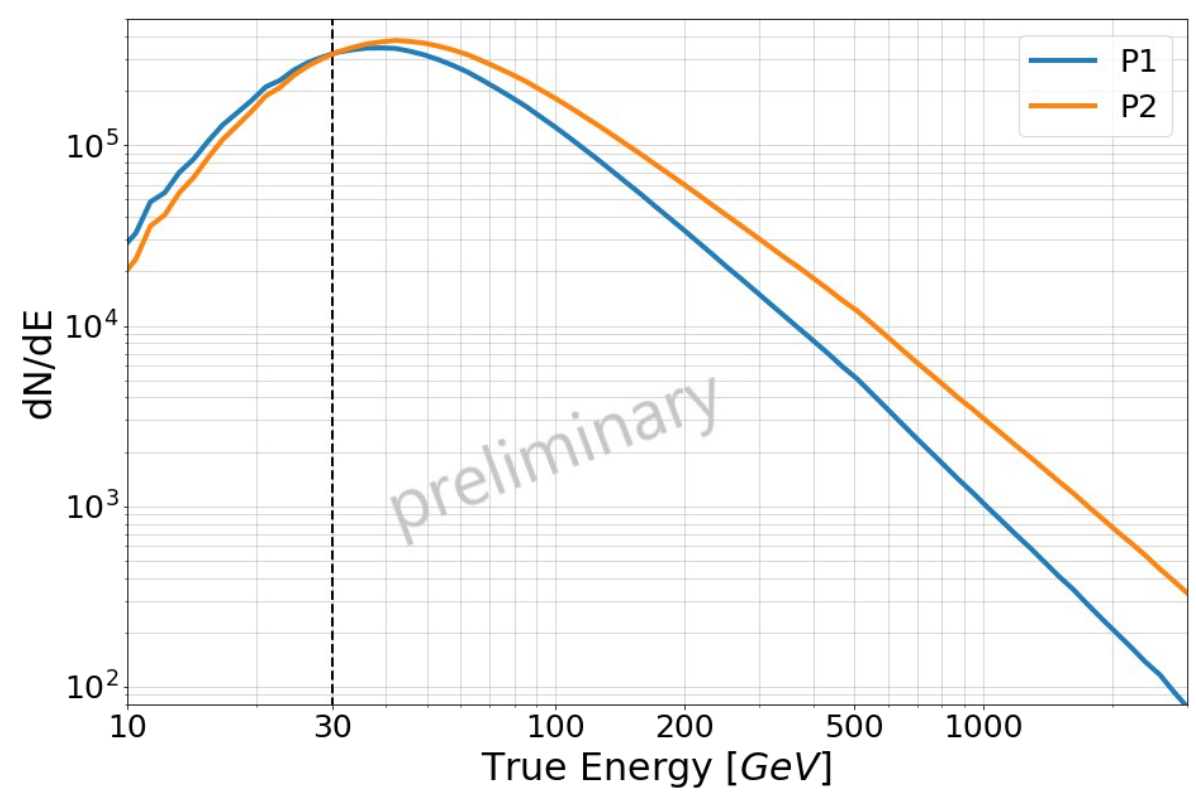

Figure 2. Total number of MC events detected by the MAGIC telescope and generated according to the flux from the Crab pulsar as a functions of true energy at phases P1 and P2. Flux values for the parameterization of the $\mathrm{P} 1$ and $\mathrm{P} 2$ are reported in Tab.1. A dashed vertical line at $30 \mathrm{GeV}$ is approximately the MAGIC energy threshold for this kind of observation.

The ratio of $\mathrm{P} 1$ to $\mathrm{P} 2$ phase amplitudes, obtained from the simulated events (in accord with Fig.2), are shown in Figure.3, left, as a function of both the true (black) and estimated (blue) energy of the Monte Carlo gamma events.
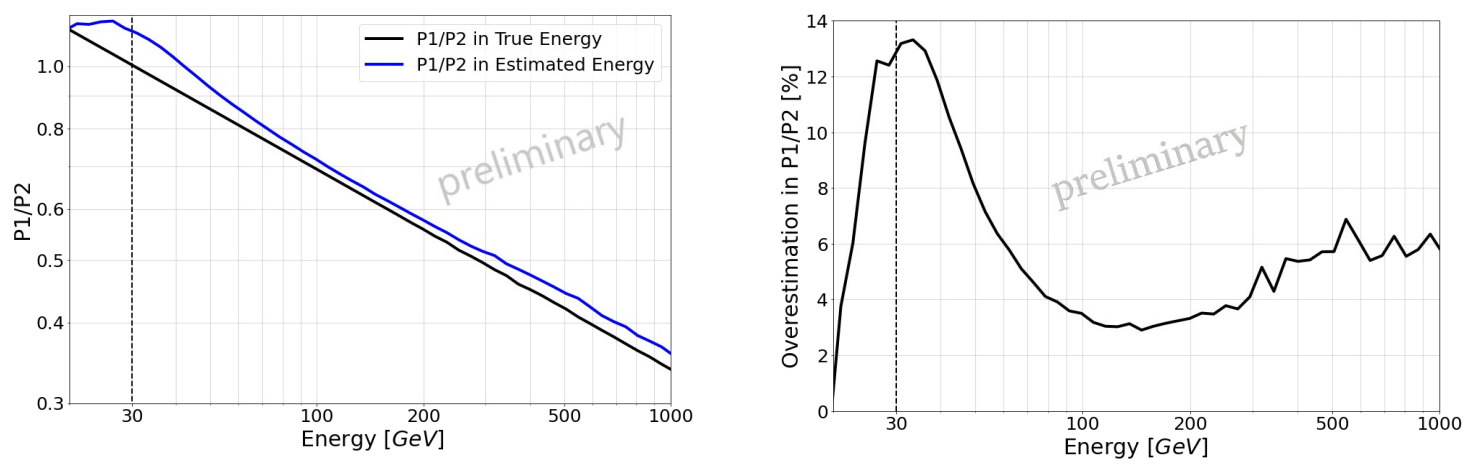

Figure 3. left: The ratio of $\mathrm{P} 1 / \mathrm{P} 2$ versus true energy (black) and estimated energy (blue); right: overestimation in P1/P2 versus energy when taking into account energy-resolution effects (i.e., the blue line of the Fig.3, left); the "hadronness" and the "theta" ${ }^{2 "}$ cuts are applied to the data (see, for example, [12].)

One can see from Fig.3, left that the P1/P2 ratio is a monotonically decreasing function of energy. When considering the true energy of the gamma, such energy decrease follows, as expected, a power-law distribution with spectral index given, by the difference of the spectral 
index of P1 and P2. Since we do not know the true energy of the gamma events observed by MAGIC, we can only obtain the energy decrease shown in blue on Fig.3, left.

In some narrow energy range the two curves are close to each other while these diverge at lower and higher energies. Obviously the estimated energy curve is higher than the true energy. On Fig.3, right we show the overestimation of P1/P2 versus energy.

At the very low energies the reduced amount of light of average showers cannot anymore produce triggers in the telescope. But due to the statistical fluctuations in the development, part of the showers penetrates deeper in the atmosphere, i.e. develops closer to the measuring telescope. As a consequence, such showers appear brighter as the average ones. The telescope can trigger on such events, i.e. on the positive fluctuations of the amount of light from the very low energy showers. As a consequence of the higher brightness, the estimated energy $\mathrm{E}_{\text {est }}$ of such events is higher than the real energy $\mathrm{E}_{\text {true }}$. One needs to correct for these when reconstructing the energy spectrum. In theory, a good estimate on the given shower maximum position would allow one to avoid or at least to reduce this effect. But the typical resolution of the maximum of shower height is poor, especially at very low energies, because of the very scarce statistics of secondary charge carriers. Therefore, as a rule such corrections are based on Monte Carlo simulations.

Please note that it belongs to the usual data analysis chain of the MAGIC collaboration to correct for the above-mentioned energy overestimation of events by applying the so-called unfolding procedure [13].

Let us have a look at Figure 1. One sort of calibration will be to search for the energy $\mathrm{E}_{*}$ where the pulse amplitude ratio of P1/P2 from the Fermi LAT is equal to that from the MAGIC data. Of course, for such study the pulse amplitudes from the data of both instruments shall be measured by applying the same cuts in the phase space around P1 and P2. One can say that at such $\mathrm{E}_{*}$ the instruments are inter-calibrated, moreover, with the absolutely calibrated reference energy. Please note that parts of the Fermi mission were absolutely calibrated before the mission as well as it monitors the efficiency during the flight.

All the written above is correct if the $\mathrm{E}_{*}$ is measured in true energy for MAGIC.

In the typical analysis of an IACT data in an almost straightforward way one obtains the estimated energy of gamma-events $\mathrm{E}_{\text {est }}$. The problem is to find the correspondence of a given $\mathrm{P} 1 / \mathrm{P} 2$ ratio from a given experiment to $\mathrm{E}_{\text {est }}$. There seem to be two obvious solutions for this; a) one can use the $\mathrm{E}_{\text {est }}$ but should pay attention that its deviation from the $\mathrm{E}_{\text {true }}$ is not too large (see more on this below), and b) use Monte Carlo simulations and apply the so-called migration matrix to the estimated energy $\mathrm{E}_{\text {est }}$ for obtaining the true energy $\mathrm{E}_{\text {true }}$. While the solution $\mathrm{b}$ ) is obvious, we would like to dwell on the seemingly simple solution a).

Now let us define $\Phi$ as the function, which for the given input $\mathrm{P} 1 / \mathrm{P} 2$ provides the corresponding energy: $\Phi(\mathrm{P} 1 / \mathrm{P} 2) \rightarrow$ E. Such function is simply the inverse of the $\mathrm{P} 1 / \mathrm{P} 2$ evolution in energy shown in the Fig.3, left.

We know from the analysis reported in Fig. 3 that there are actually two $\Phi$ functions, $\Phi_{\text {ideal }}$ for the ideal case in which we have a perfect energy resolution (black line on Fig.3, left and $\Phi_{\text {real }}$ for the real case in which migration matrix effects are taken into account (blue line on Fig.3, left ). 
Unfortunately, from the experiment we know only the $\Phi_{\text {real }}$. When calibrating the energy from the observed $\mathrm{P} 1 / \mathrm{P} 2$ ratio, we therefore introduce an energy overestimation due to the difference between $\Phi_{\text {real }}$ and $\Phi_{\text {ideal }}$. Such overestimation is shown in Fig.4, where on the x-axis we show $\Phi_{\text {real }}(\mathrm{P} 1 / \mathrm{P} 2)$, while on the y-axis the overestimation, defined as

$$
\left[\Phi_{\text {real }}(\mathrm{P} 1 / \mathrm{P} 2)-\Phi_{\text {ideal }}(\mathrm{P} 1 / \mathrm{P} 2)\right] / \Phi_{\text {real }}(\mathrm{P} 1 / \mathrm{P} 2)
$$

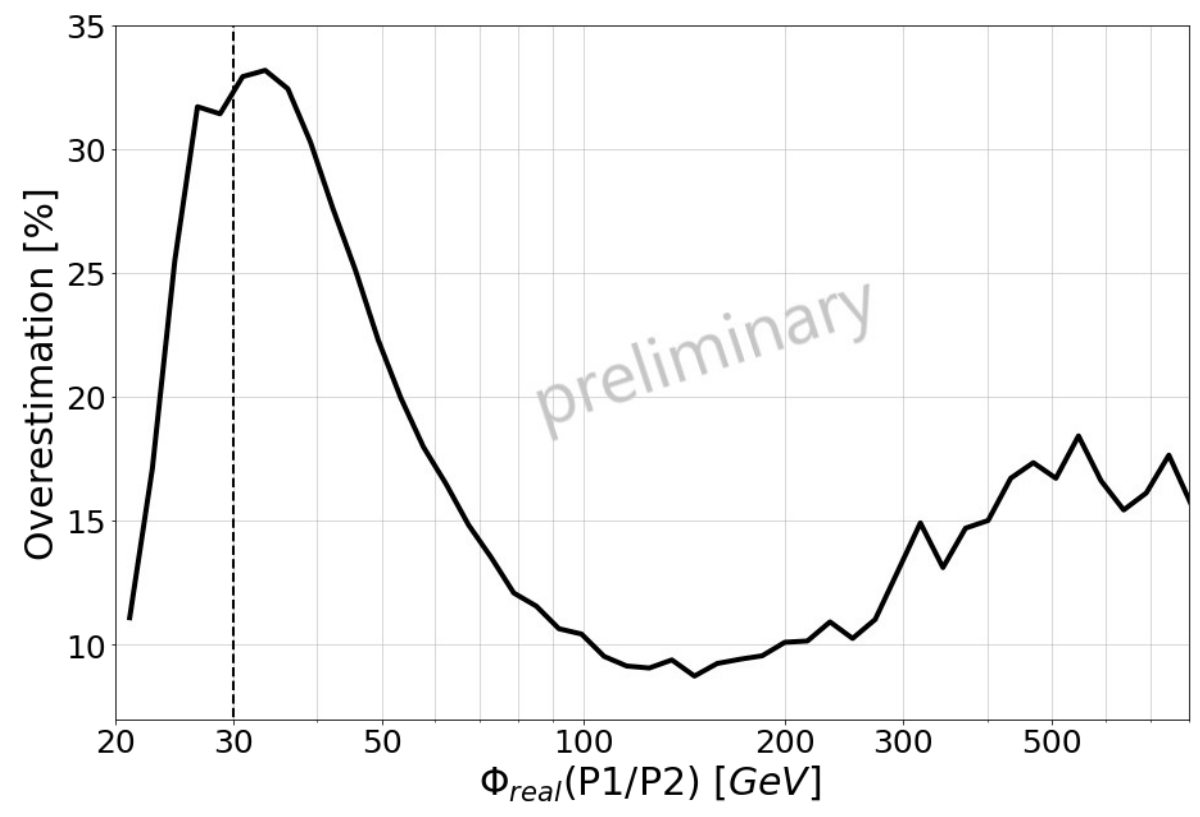

Figure 4. Energy overestimation, as defined in Eq.1, versus the energy obtained from the $\Phi_{\text {real }}(\mathrm{P} 1 / \mathrm{P} 2)$.

One can see that the energy overestimation stays below $20 \%$ for energies $\geq 53 \mathrm{GeV}$, till $\sim 800 \mathrm{GeV}$, and it is on the level of $\sim 10 \%$ in the narrow energy range $100-200 \mathrm{GeV}$. The overestimation degrades for energies $\leq 50 \mathrm{GeV}$, and it is becoming an effect of $33 \%$ close to the threshold of the telescope at about $30 \mathrm{GeV}$, see Fig.4.

\section{Conclusions}

We studied the possibility of estimating the threshold of an IACT telescope from the measured ratio of P1/P2 phase pulses of the Crab Nebula by a simple, independent on Monte Carlo simulations method. The MAGIC IACT data on P1/P2 ratio has been compared with the absolutely calibrated Fermi-LAT data. The results show that one can pinpoint the operational energy range from the given $\mathrm{P} 1 / \mathrm{P} 2$ ratio with accuracy, shown in Fig.4. The energy overestimation is below $10 \%$ for the energy range (100-200) $\mathrm{GeV}$, it is below $15 \%$ from $\sim(65-$ 400) $\mathrm{GeV}$, while for even higher energies it increases to the level $\sim 18 \%$. Close to the threshold of the telescope at around $30 \mathrm{GeV}$ the simple estimate overestimates the energy by $\sim 33 \%$. 


\section{Acknowledgements}

We kindly acknowledge the interest and support of the members of the MAGIC collaboration during the course of this study.

\section{References}

[1] Abdo, A.A., Ackermann, M., Ajello, M., et al, Science, 331, 6018 (2011) 739

[2] Tavani, M., Bulgarelli, A., Vittorini, V., et al, Science, 331, 6018 (2011) 736

[3] van Scherpenberg, J., Mirzoyan, R., Vovk, I., et al, 36th ICRC (2019) Madison, WI, U.S.A.; arXiv: 1909.04356v1

[4] Thompson, D. J., “Gamma Ray Pulsars”, arXiv:astro-ph/0312272 (2003)

[5] The MAGIC Collaboration: Albert, J., Aliu, E., Anderhub, H., et al, ApJ 674 (2008) 1037

[6] The MAGIC Collaboration: Aliu, E., Anderhub,, H., Antonelli, L.A., et al, Science 322, (2008) 1221

[7] The MAGIC Collaboration: Aleksi' c, J., Alvarez, E.A., Antonelli, L.A., et al, ApJ 742:43 (14pp) (2011)

[8] The Veritas Collaboration: Aliú E., Arlen, T., Aune, T., et al, Science 334, 69 (2011)

[9] The MAGIC Collaboration: Aleksi' c J., Alvarez, E. A., Antonelli, L. A., et al, $A \& A 540$ A69 (2012)

[10] The MAGIC Collaboration: Aleksi' c, J., Ansoldi, S., Antonelli, L.A., et al, A\&A 565, L12 (2014)

[11] The MAGIC Collaboration: Ansoldi, S., Antonelli, L.A., Antoranz, P., et al, $A \& A 585$ A133 (2016)

[12] Ceribella, G., "Insights into the 10-100 GeV gamma-ray emission of pulsars from extensive observations of MAGIC", PhD thesis, Technical University of Munich, 2021.

[13] The MAGIC Collaboration: Albert, J., Aliu, E., Anderhub, H., et al, Nucl. Instr. Meth. Phys. Res. A, 583 (2007) 494 